\title{
Stable models and an alternative logic programming paradigm
}

\author{
Victor W. Marek, Mirostaw Truszczynski \\ Department of Computer Science \\ University of Kentucky \\ Lexington, KY 40506-0046 \\ \{marek, mirek\}@cs.engr.uky.edu
}

\begin{abstract}
In this paper we reexamine the place and role of stable model semantics in logic programming and contrast it with a least Herbrand model approach to Horn programs. We demonstrate that inherent features of stable model semantics naturally lead to a logic programming system that offers an interesting alternative to more traditional logic programming styles of Horn logic programming, stratified logic programming and logic programming with well-founded semantics. The proposed approach is based on the interpretation of program clauses as constraints. In this setting programs do not describe a single intended model, but a family of stable models. These stable models encode solutions to the constraint satisfaction problem described by the program. Our approach imposes restrictions on the syntax of logic programs. In particular, function symbols are eliminated from the language. We argue that the resulting logic programming system is well-attuned to problems in the class NP, has a well-defined domain of applications, and an emerging methodology of programming. We point out that what makes the whole approach viable is recent progress in implementations of algorithms to compute stable models of propositional logic programs.
\end{abstract}

\section{Introduction}

Stable model semantics appeared on the logic programming scene in the late $80 \mathrm{~s}$ in an effort to provide an understanding of programs with negation. Since it was first proposed by Gelfond and Lifschitz GL88, it was regarded by logic programming community with a dose of reserve and unease. It was intuitively felt that the stable model semantics properly deals with negation and some formal evidence supporting this intuition was established. At the same time, the stable model semantics did not fit into a standard paradigm of logic programming languages. While standard approaches assign to a logic program a single "intended" model, stable model semantics assigns to a program a family (possibly empty) of "intended" models. Further, in the presence of function symbols, Horn logic programs can specify any recursively enumerable set, while stable model semantics increases the expressive power of logic programs well beyond 
acceptable notions of computability. Finally, the SLD-resolution, the true bread-and-butter of logic programmers and the heart of control mechanisms behind standard logic programming implementations, seems to be inappropriate for the stable model semantics.

As a consequence of these difficulties in reconciling the stable model semantics with a traditional paradigm of logic programming, the stable model semantics received relatively less attention from the logic programming community than other semantics proposed for programs with negation such as perfect model semantics for stratified programs and well founded semantics.

In this paper we argue that rather than to try to resolve these inconsistencies and force stable model semantics into a standard logic programming mold (this effort most likely is doomed to failure), a change of view is required. Therefore, we propose a perspective on the stable model semantics that departs from several basic tenets of logic programming. At the same time, this perspective leads to a computational system very much in the general spirit of logic programming. The system is declarative, retains the separation of logic from control, has a well-defined domain of applications and emerging programming methodology. We refer to this version of logic programming as stable logic programming (or, SLP, for short).

There are several key elements to the view on the stable model semantics that we describe here. First, we restrict the syntax by disallowing function symbols. Thus, the syntax of SLP is the same as the syntax of DATALOG with negation. The restriction of the syntax has significant effect on the expressive power of programs. In particular, it curtails the ability to use recursion.

Second, we view a program as specifying a collection of models rather than a single model. Thus, SLP seems to be especially well suited for all these problems where solutions are subsets of some universe, as each solution can be modeled by a different stable model. Many combinatorial and constraint satisfaction problems fall into this category.

The restricted syntax (limiting the use of recursion) and the shift in the semantics (programs specify a collection of models rather than a single model), change the way in which we interpret and design programs. Programs are interpreted as sets of constraints. Clauses represent individual constraints on objects of interest rather than recursive definitions of these objects. Hence, a different approach to programming is needed. Objects that in Horn logic programming would be represented as terms and defined recursively, in SLP are represented as different stable models and defined in terms of constraints.

Finally, although the SLP follows the basic logic programming tenet of "uniform control", the control used in SLP is different. Instead of SLD resolution used in Horn Logic programming, a backtracking search for stable models is used.

While (Horn) logic programming is well attuned to the concepts of Turing computability, recursively enumerable sets and partial recursive functions, SLP is related to a much more narrow class of problems. As we point out, all decision problems that are in NP can be solved within the paradigm of SLP. Moreover many (possibly all) search problems, whose decision versions are in NP, can be solved with SLP programs, too. While NP is much smaller than the class of r.e. sets, it still covers a wide collection of important computational problems including many combinatorial optimization and constraint satisfaction problems. This is one of the main reasons why we believe that SLP can evolve into a useful computational tool.

Recently, the idea of using logic programs with restricted syntax and nonstandard semantics was discussed in several papers. Niemelä [Nie98], in a closely related work, proposed function 
symbol-free logic programming with stable model semantics as a vehicle to process constraint satisfaction problem. Second, Cadoli and others CP98, proposed the use of DATALOG programs without negation and with the semantics of parallel circumscription as a tool for solving decision problems in NP. Finally, in CMMT98 we study a similar use of default logic (a formalism extending SLP) as a programming environment for solving decision problems from the class $\Sigma_{2}^{P}$.

All these projects are supported by algorithms to process logic programs and by their implementations. Niemelä and Simons NS95, NS96] developed a system, smodels, to compute stable models of logic programs. Algorithms to process DATALOG programs under parallel circumscription were presented in CPSV98. A system DeReS to process a wider class of programs - default theories - is described in [CMT96, CMMT98]. The emergence of implementations is the main reason why these alternative logic programming systems are becoming viable computational tools.

This paper is organized as follows. In the next section we provide a perspective of Horn logic programming, pointing to those of its features that are responsible for its expressivity. We discuss the effects of the negation operator in logic programming and show how it increases the expressivity beyond the currently accepted bounds. In Section 6 we formally introduce stable logic programming (SLP). Next, we study the expressive power of SLP, showing that the applicability of SLP is attuned to the class NP. In Section 6 we note the limitations in using recursion in SLP and show that SLP programs should be interpreted as descriptions of sets of constraints. Consequently, the methodology of programming with SLP is different from that of ordinary logic programming. We discuss several examples and show how SLP programs that solve them can be developed. We discuss the issue of uniform control associated with SLP in Section 7. Conclusions and a "road map" for the future complete the paper.

\section{Horn logic programming}

The idea to use logic as a computational mechanism can be traced back to the Herbrand's analysis of the effectivity of proofs in first-order logic [Her30]. In this work Herbrand discovered a unification algorithm, one of the basic constructions behind the present-day automated deduction and logic programming.

Transforming logic into a viable programming tool required two additional crucial steps. First of them was the introduction of the resolution by Robinson Rob65 with unification as one of its key components. Over the years resolution was extensively studied [CL73, Lov78, MW88] and gained in stature as one of the most successful techniques of automated reasoning.

The second key step was to narrow down the focus of automated deduction to the class of Horn theories and even more specifically, to the class of definite Horn theories. A definite Horn clause is a formula of the form

$$
q_{1}(\vec{X}) \wedge \ldots \wedge q_{k}(\vec{X}) \Rightarrow p(\vec{X})
$$

(written $p(\vec{X}) \leftarrow q_{1}(\vec{X}), \ldots, q_{k}(\vec{X})$ by the logic programming community), where $q_{i}$ and $p$ are atoms of the language. A definite Horn theory (a Horn logic program) is a finite theory consisting of definite Horn clauses.

Each definite Horn theory $P$ is consistent and possesses a least Herbrand model, $\operatorname{LM}(P)$. This least model provides a natural and intuitive semantics for definite Horn theories and leads 
to a natural concept of computability. We say that a finite definite Horn theory $P$ specifies a subset $X$ of the Herbrand universe of $P, H U(P)$, if for some predicate symbol $p$ occurring in $P$ we have:

$$
X=\{t \in H U(P): p(t) \in L M(P)\} .
$$

One of the fundamental results underlying the area of logic programming is that every recursively enumerable set can be specified by a definite Horn theory [Smu68, AN78]. This result shows that definite Horn theories are as expressive as Turing machines and precisely capture the concept of Church-Turing computability.

The area of logic programming was born in early 70s when it was realized that the elements of the set $\{t \in H U(P): p(t) \in L M(P)\}$ can actually be computed. Namely, a special form of resolution, the SLD-resolution [Kow74, AvE82], and the so-called lifting lemma [Llo84 allow us to compute ground terms $t$ (more generally, ground substitutions $\sigma$ ) such that $p(t)$ (or $p(\vec{X} \sigma)$ ) belongs to the least Herbrand model of a definite Horn theory.

Availability of this uniform control mechanism, SLD-resolution, is the key aspect of Horn logic programming. Horn programs, that is, definite Horn theories, need only to specify definitions and properties of objects and domains of interest. The programmer no longer needs to specify the exact way in which to perform the computation. The control is provided by the mechanism of the SLD resolution. This feature of logic programming, the separation of logic ("what" part) from control ("how" part), was and still remains one of the most attractive features of logic programming. It allows the programmers to focus only on the logic of the problem and frees them from the burden of specifying the control. Consequently, it carries with itself a promise of easier code development, facilitates modular design of software and eases the problems of program verification] [Kow79]. It is due to the separation of logic from control that logic programming is often classified as a declarative programming system.

Function symbols are critical for the Horn logic programming. The presence of function symbols in the language, especially of the list constructor [·], allows the programmer to encode hereditarily finite higher-order objects as terms of the language. Consequently, logic program clauses can be used to describe recursive definitions of higher-order objects. Modeling the recursive definitions within logic programs is responsible for the expressive power of logic programming and became one of its most important and most common programming techniques.

The situation changes drastically if function symbols (in particular, [.]) are not available. This formalism was extensively studied as a possible query language by the database community and is known there as DATALOG (see, for instance, Ull88). With no function symbols, the Herbrand universe of finite Horn programs is finite. Thus, the ability to represent higherorder objects in DATALOG is significantly restricted. Similarly, many recursive definitions can no longer be modeled by DATALOG clauses (those which describe how more complex objects of higher order can be constructed from simpler ones). Consequently, the expressive power of finite DATALOG programs is very limited. They can only express a proper subset of all polynomial-time computable queries ACY91. Thus, it is the presence of function symbols in the language that is responsible for the expressive power of Horn logic programming.

We will now summarize some of the key features of Horn logic programming.

\footnotetext{
${ }^{1}$ This expectation did not entirely materialize. Peculiarities of logic programming implementations, including subtleties of search space pruning and its side effects resulted in a paradigm not easily accepted by real-life programmers.
} 
1. The existence of a single intended model (a least Herbrand model) yields the semantics for Horn logic programs and the notion of computability. Horn programs compute extensions of predicates in the least model.

2. Function symbols in the language allow the programmer to encode higher-order objects as terms and represent recursive definitions of these objects by means of Horn clauses.

3. Horn programs can specify any recursively enumerable set. Thus, Horn logic programming precisely captures the commonly accepted notion of computability.

4. Horn logic programming is declarative due to the separation of logic, represented by Horn programs, from uniform control, represented by the SLD resolution.

\section{$3 \quad$ Negation in logic programming}

As observed above, from the point of view of the expressive power, the Horn logic programming is as powerful as any programmer might want it to be - it captures recursively enumerable sets. However, as a declarative system, it was not quite satisfying. The ability to describe intuitive declarative specifications of objects to be computed was significantly hampered by disallowing the negation operator from the bodies of clauses. This was recognized very early on in the development of the field. In particular, the negation operator has been available in PROLOG since its creation [CKPR73] and extensions of Horn logic programming with negation in the bodies of program clauses were studied since mid 1970s.

In fact, the effort to extend Horn logic programming by allowing the negation operator in the bodies was among the strongest driving forces behind the development of the area in the past 25 years. The task is far from straightforward as adding negation implies that the existence of a unique least model (one of the fundamental features of the Horn logic programming paradigm) is no longer guaranteed.

Proposals to address the problem can be divided into two classes. Proposals of the first type attempt to salvage the notion of a single intended model at a cost of narrowing down the class of programs or weakening the semantics. Apt, Blair and Walker ABW88 introduced the notion of stratification, a syntactic restriction on logic programs with negation. They assigned to each stratified program a single intended model, a perfect model. In another approach, van Gelder, Ross and Schlipf VRS91 assigned to an arbitrary program a single intended 3-valued model, a well-founded model. These two proposals are still very much in the spirit of the Horn logic programming paradigm. Namely, in each of these approaches, a program specifies extensions of predicates in a single intended model - perfect or well-founded, respectively. Moreover, suitably modified versions of resolution were proposed as a uniform processing mechanisms RRS ${ }^{+} 95, \mathrm{RRS}^{+} 97$.

However, both these approaches lead to problems with excessive expressive power. Apt and Blair [AB90] proved that stratified programs with finite number of strata specify precisely arithmetic sets. This means that they are expressive beyond what is at present considered computable. Since the well-founded model coincides with perfect model for stratified programs, the same result applies to well-founded semantics 2 . In fact, perfect models of locally stratified

\footnotetext{
${ }^{2}$ In particular, the programmer that uses a logic programming environment based on the well-founded seman-
} 
programs [Prz88] specify an even bigger class of sets - the class of hyperarithmetic sets BMS95. The same class of sets is specified by programs for which the well-founded model is total while, in general, well-founded semantics specifies the class of $\Pi_{1}^{1}$ sets Sch95.

The common idea behind the proposals of the second type was to distinguish among all models of a program a collection of intended models rather than a single one. The class of supported models was introduced by Clark Cla78] and, almost ten years later, Gelfond and Lifschitz GL88 fine-tuned Clark's approach and defined the class of stable models as candidates for intended models of logic programs with negation.

Our goal in this paper is to present a perspective of logic programming with stable model semantics and, thus, from now on we will focus on stable models only. Let us briefly recall the definition of a stable model (for a detailed treatment, the reader is referred to [MT93). Let $P$ be a logic program and let $P_{g}$ be the grounding of $P$. A subset $M$ of the Herbrand base of $P$ is a stable model of $P$, if $M$ coincides with the least model of the reduct $P_{g}^{M}$ of $P_{g}$ with respect to $M$. The reduct $P_{g}^{M}$ is the program obtained from $P_{g}$ by removing from $P_{g}$ all clauses containing in the body a literal of the form $\operatorname{not}(p)$, for some $p \in M$, and by removing literals of the form $\operatorname{not}(p)$ from all of the remaining clauses. Directly from this definition one can derive the following fundamental properties of stable models. First, every stable model of a logic prgram $P$ is, indeed, a model of $P$. Furthermore, every stable model of $P$ is a minimal model of $P$ and a supported model of $P$. Finally, the family of stable models of a program form an antichain.

The stable model semantics, from the day it was proposed, was the subject of some controversy. On one hand, it was commonly accepted that stable models provide the right semantics for logic programming with negation. There is an abundance of evidence to support this claim. For instance, it is known that the stable model semantics coincides with the least model semantics on definite Horn programs. For stratified logic programs it coincides with the perfect model semantics. Further, when the well-founded model is total, it defines a unique stable model VRS91]. Finally, the well-founded model is the least three-valued stable model [Prz90]. In addition, as demonstrated in MT89, BF91, Kam97, logic programming with the stable model semantics is closely related to default logic of Reiter [Rei80, a commonly accepted formalism for knowledge representation.

On the other hand, it was not clear how to reconcile the stable model semantics with the paradigm of Horn logic programming, as presented in Section 2. Three problems are: lack of a single intended model (the notion of specification given by (1) requires modifications), increase in the expressive power beyond the accepted limits of computability, and inadequacy of resolution-based control.

The first problem can, to some degree, be overcome by means of the so called skeptical semantics. Under this semantics, a ground atom is entailed by the program if it is true in all of its stable models. We say that a logic program with negation $P$ specifies a subset $X$ of $H U(P)$, if for some predicate symbol $p$ occurring in $P$ we have:

$$
X=\left\{t \in H U(P): P \models_{\text {skeptical }} p(t)\right\} .
$$

However, at this point, problems with the expressive power reappear. Indeed, stratified pro-

tics (for instance XSB) must understand that some programs specify complex sets for which computation will not terminate. The non-termination occurs in Horn logic programming as well, but a more complex semantics adds an additional layer of complexity to programmer's task. 
grams have unique stable model, thus skeptical semantics coincides with perfect model semantics for such programs. Consequently the results on the expressivity of perfect semantics for stratified programs apply in this situation. Moreover, the problem to decide the existence of a stable model of a finite logic program is $\Sigma_{1}^{1}$-complete, while the problem to decide the membership in all stable models is $\Pi_{1}^{1}$-complete [MNR92]. Thus, both problems are far beyond accepted notions of computability.

There is one additional complication. Due to the complexity, no form of resolution can be applicable in the general case of skeptical semantics, without drastic restrictions on the syntax of programs. Whereas in the case of well-founded semantics conditions limiting the complexity are known and resolution-based systems for well-founded semantics were developed [RRS ${ }^{+} 95, \mathrm{RRS}^{+} 97$, in the case of skeptical semantics such results have yet to be established.

\section{$4 \quad$ Stable logic programming}

The difficulty of fitting logic programming with stable model semantics into the paradigm of Horn logic programming, combined with an intuitive appeal of stable models, makes us believe that the place of stable model semantics in logic programming must be reexamined. To this end, we propose below an alternative paradigm to that of the Horn logic programming, a paradigm consistent with the properties of stable models. We will refer to it as stable logic programming (or, SLP for short).

While stable logic programming is in many aspects different from Horn logic programming, at the most general level it shares with it the key feature of separation of logic from control. Consequently, as other logic programming formalisms, stable logic programming is declarative. The programmer specifies the problem at hand as a logic program. This program is then processed by a uniform control mechanism, thus solving the original problem. The differences are in the syntax and semantics. These differences affect the control mechanism (it is no longer the SLD resolution), as well as the expressive power of stable logic programming and the corresponding programming methodology.

We will now specify stable model programming and discuss these differences in more detail. First, we will restrict the syntax since, as we saw earlier, without any restrictions the expressive power gets out of hand. Trivially, the negation operator must remain in the language (there is no need for the stable model semantics without it). The other major source of complexity, function symbols, must be eliminated, however. Indeed, even under a restriction of stratification, in the presence of both the negation operator and function symbols in the language, the complexity grows beyond the limits of computability [AB90]. Thus, in stable logic programming we adopt the language of logic programming that consists of denumerable collections of constant, variable and predicate symbols. In addition, we will allow for the negation operator to appear in the bodies of program clauses. Finite programs in this language will be referred to as SLP programs.

Due to the presence of negation, the existence of a least Herbrand model is no longer guaranteed. The semantics of SLP programs will be defined in terms of their stable models. Before we address this issue in more detail, let us observe that the formalism of finite function symbol-free logic programs with negation, was extensively studied by the database community. The formalism is often referred to as DATALOG $\urcorner$ and several semantics for DATALOG $\urcorner$ were studied. Stratified version of DATALOG $\urcorner$ with perfect model semantics and with well-founded 
semantics received particular attention AHV95. At the same time, DATALOG $\urcorner$ with stable model semantics has never drawn any significant interest in database community, mostly due to the fact that under the stable model semantics there is no guarantee of a single intended model that might be used to determine an answer to a query stated as a DATALOG $\urcorner$ program. Skeptical stable semantics was not regarded as quite satisfactory either as the set of atoms entailed under this semantics is not a model of a DATALOG $\urcorner$ query.

The lack of a single intended stable model, perceived as a problem by the logic programming and database communities, plays the key role in stable logic programming. Under the stable model semantics, a finite SLP program can be viewed as a specification of a finite family of finite sets. Namely, we say that a finite SLP program $P$ specifies a family of sets $\mathcal{X}$ if for some $k$-ary predicate $p$,

$$
\mathcal{X}=\left\{\left\{\left(c_{1}, \ldots, c_{k}\right) \in H U(P): p\left(c_{1}, \ldots, c_{k}\right) \in M\right\}: M \text { is a stable model of } P\right\} .
$$

This notion of specification is the counterpart, in the case of SLP, of the notion of specification given by (1) in the case of Horn logic programming. It allows the programmer, without resorting to function symbols, to write logic programs that specify second-order objects which, in Horn logic programming, would be encoded by means of terms involving the [.] operator. Thus, as we will state it more formally later, multiple intended models allow us to recover in SLP some of the expressive power of logic programming lost by eliminating function symbols from the language.

In addition, the notion of specification given by (2) suggests that SLP programs are very well suited to represent problems whose solutions are finite families of finite sets. For instance, a hamiltonian cycle in a directed graph is a set of edges, that is, a set of pairs of vertices. The collection of all hamiltonian cycles of a graph is then a collection of sets of pairs, that is an object of the form given by (2). In Section 6, we will exhibit explicit SLP programs that represent the hamiltonian cycle problem.

\section{Expressive power of SLP}

Following Garey and Johnson GJ79, we define a search problem, $\Pi$, to consist of a set of finite instances, $D_{\Pi}$. Further, for each instance $I \in D_{\Pi}$, there is a finite set $S_{\Pi}(I)$ of all solutions of $\Pi$ for the instance $I$. An algorithm solves a search problem if for each instance $I \in D_{\Pi}$ it returns the answer "no" when $S_{\Pi}(I)$ is empty, and any solution $s \in S_{\Pi}(I)$, otherwise. Notice that all decision problems can be viewed as special search problems: for every instance $I$ of a decision problem, define $S_{\Pi}(I)=$ "yes" $\}$, if $I$ is a "yes" instance of $\Pi$, and $S_{\Pi}(I)=\emptyset$, otherwise. Notice also that with each search problem one can associate a decision problem: given an instance $I \in D_{\Pi}$, decide whether $S_{\Pi}(I)=\emptyset$.

Several interesting search and decision problems can be associated with stable logic programming. Consider a finite SLP program $P$. Clearly, the Herbrand universe, the Herbrand base and the grounding of $P$ are all finite. Consequently, stable models of $P$ (if exist) are finite, too. It follows that the problem to compute, given a finite SLP program, its stable models is a search problem. Another search problem of interest is, given a finite SLP $P$ and an element $a$ of the Herbrand base $H B(P)$, to compute a stable model of $P$ containing $a$. An associated decision problem asks for the existence of stable models of a finite SLP program $P$. Other 
related decision problems ask whether a given element $a$ of the Herbrand base $H B(P)$ belongs to some (or all) stable models of $P$.

To understand the expressive power of SLP, we need to study which search and decision problems can be reduced to search and decision problems associated with SLP. We will first consider the class of decision problems and restrict, for a moment, to propositional programs only. The following theorem MT91 plays a key role in our discussion.

Theorem 5.1 The problem to decide whether a finite propositional logic program has a stable model is NP-complete.

Theorem 5.1 implies that for every decision problem $\Pi$ in the class NP and for every instance $I \in D_{\Pi}$, there is a propositional program $P_{\Pi}^{I}$ such that

(1) $P_{\Pi}^{I}$ can be constructed in time polynomial in the size of $I$, and

(2) $\Pi$ has a solution for $I$ if and only if $P_{\Pi}^{I}$ has a stable model.

Thus, any decision problem $\Pi$ in NP can be solved by a uniform control mechanism of deciding existence of stable models of logic programs. Given an instance $I$ for which $\Pi$ must be decided, one may encode $\Pi$ and $I$ as a propositional logic program $P_{\Pi}^{I}$ (that can be constructed in time polynomial in the size of $I$ ) and decide whether $I$ is a "yes" instance of $\Pi$ by deciding whether $P_{\Pi}^{I}$ has a stable model. Moreover, any decision problem that can be decided in this way is in NP.

Consider now a search problem $\Pi$. Assume that for every instance $I \in D_{\Pi}$, there is a propositional program $P_{\Pi}^{I}$ satisfying the condition (1) and a stronger version of the condition $(2)$ :

$\left(2^{\prime}\right)$ there is a polynomially computable one-to-one function $\operatorname{sol}_{\Pi}^{I}$ from the class of stable models of $P_{\Pi}^{I}$ to $S_{\Pi}(I)$.

Search problems of this type can be solved in a similar way to the one described earlier for the case of decision problems. Given an instance $I \in D_{\Pi}$, one constructs the program $P_{\Pi}^{I}$, finds its stable model $s$, computes $\operatorname{sol}_{\Pi}^{I}(s)$ and returns it as a solution to $\Pi$ for $I$. If no stable model exists, the answer "no" is returned.

To the best of our knowledge, it is an open problem to characterize the class of search problems for which this approach can be used, that is, the class of search problems $\Pi$ for which programs $P_{\Pi}^{I}$ satisfying conditions $(1)$ and $\left(2^{\prime}\right)$ can be found. We saw that all decision problems in the class NP can be solved in this way. Furthermore, all search problems whose associated decision problems are in NP, that we considered so far, also can be dealt with in this way (some of the encodings will be discussed later in the paper).

The approach presented above relies on encodings of decision and search problems as problems involving existence or computation of stable models of propositional programs. It is not entirely satisfactory as different programs $P_{\Pi}^{I}$ are needed for each instance $I$ of a problem or, to put it differently, the logic is not separated from data. We will now present yet another possible approach that takes advantage of variables in the language.

Consider a search problem $\Pi$. Assume that there exist:

(1) an effective encoding $e d b_{\Pi}$ under which every instance $I \in D_{\Pi}$ is represented as a database under some, fixed for all instances from $D_{\Pi}$, relational database scheme, 
(2) a finite SLP program, $P_{\Pi}$, such that for every instance $I$, that there is a polynomially computable one-to-one function $s o l_{\Pi}^{I}$ from the class of stable models of $e d b_{\Pi}(I) \cup P_{\Pi}$ to $S_{\Pi}(I)$.

Then, $\Pi$ can be solved for an instance $I$ by first constructing the program $e d b_{\Pi}(I) \cup P_{\Pi}$, then by finding its stable model $s$ and, finally, by reconstructing from $s$ a solution $\operatorname{sol}_{\Pi}^{I}(s)$.

This approach is more elegant and more in the spirit of standard programming. In this approach, $P_{\Pi}$ can be regarded as a program (logic) for solving the problem $\Pi$, and the database $e d b_{\Pi}(I)$ can be viewed as data. Thus, there is a clear separation of logic (uniform over all possible instances to the problem) and data (encodings of problem instances).

We say that a search problem can be solved by a uniform SLP program if there exist an encoding $e d b_{\Pi}$ and a program $P_{\Pi}$ satisfying (1) and (2). As in the case of two earlier approaches, the question is which search problems can be solved by a uniform SLP program. The following strengthening of Theorem 5.1 was first proved in Sch95.

Theorem 5.2 A decision problem can be solved by a uniform SLP program if and only if it is in the class NP.

Thus, for decision problems we have a complete answer. The problem remains open for arbitrary search problems. Let us point out, though, that all search problems, whose associated decision problems are in NP, that we considered so far, can be so solved.

To summarize, due to the absence of function symbols, the expressive power of stable logic programming is restricted as compared to Horn logic programming. However, due to the use of negation and the stable model semantics, some of the lost expressive power is recovered. SLP can capture all decision problems in NP and many (perhaps all) search problems whose decision versions are in NP.

\section{Recursion versus constraints}

The restrictions in the syntax of SLP, the change in the semantics and, consequently, the change in the notion of specification (from (1) to (2)) requires a different approach to programming. Perhaps most importantly, the use of recursion is severely restricted. A limited version of recursion is still available. Namely, recursive definitions of predicates (or rather of sets that are their extensions) can be modeled by SLP clauses. For instance, the following SLP clauses define the transitive closure of a relation $r e l$

$$
\begin{aligned}
& t c(X, Y) \leftarrow \operatorname{rel}(X, Y) \\
& t c(X, Y) \leftarrow t c(X, Z), \operatorname{rel}(Z, Y)
\end{aligned}
$$

However, without function symbols to build terms representing higher-order objects, it is far from clear how a clause could capture such recursive definitions that specify how more complex objects are constructed from simpler ones. For instance, consider the following HLP program:

$$
\begin{aligned}
& \operatorname{clique}([X]) \leftarrow \operatorname{vertex}(X) \\
& \operatorname{clique}([Y \mid X]) \leftarrow \operatorname{clique}(X), \operatorname{allconnected}(Y, X)
\end{aligned}
$$


We assume here that the predicate allconnected $(Y, X)$ is defined so that to succeed precisely when vertex $Y$ is connected by an edge to all vertices on list $X$, and when $X$ has no repetitions. The program consisting of the definition of allconnected, of clauses (1) and (2) and of the description of a graph $G$ in terms of facts specifying the extensions of the predicates vertex and edge, computes all cliques in $G$. Notice that each time clause (2) is used in computation, it produces a longer list (more precisely, each iteration of the one-step operator associated with this program generates new ground terms that need to be included in the extension of the predicate clique. The capability of growing the set of available ground terms is both a strength and a weakness of HLP. It allows to code all hereditarily finite sets but, at the same time, makes it possible to write programs that do not terminate.

This phenomenon does not occur in SLP - the available constants are prescribed from the beginning and no new terms can be built of these constants as there are no function symbols that could accomplish this. At the same time, as we saw earlier, SLP is expressive enough to specify some higher-order objects. Namely, an SLP program specifies the collection of its stable models (or, as we defined in (2), collections of extensions of predicates in stable models). Clauses of the program do not represent recursive definitions of individual stable models but act as constraints. More specifically, a ground clause

$$
C=p \leftarrow q_{1}, \ldots, q_{m}, \operatorname{not}\left(r_{1}\right), \ldots, \operatorname{not}\left(r_{n}\right)
$$

expresses the following constraint: at least one $q_{i}$ does not belong to a putative stable model $M$, or at least one $r_{j}$ belongs to $M$, or $p$ belongs to $M$. In other words, $M$ must be a model of the clause $C$ treated as a propositional formula. Moreover, in a crucial difference with propositional logic, stable logic programming adds to this constraint also a preferred way to compute sets that satisfy it: in order to enforce the constraint, once all $q_{i}$ are computed and none of $r_{j}$ was established, $p$ is added to the set rather than some $q_{i}$ eliminated or some $r_{j}$ added.

Here lies the key difference between Horn logic programming and stable logic programming. To specify a higher-order object in Horn logic programming, the programmer models the object as a term and represents in a program a recursive definition of the object. To specify a secondorder object in SLP, the programmer thinks of the object as a stable model of a program and constructs the program by modeling a definition of the object expressed in terms of constraints. This feature makes SLP especially well suited to deal with constraint satisfaction problems, a point made in Nie98].

An important issue raised by our discussion is how to represent constraints by SLP clauses. We will now present one such technique of adding selection clauses (in the case of default logic, this techniques is discussed in CMMT98).

Many applications specify objects of interest to a problem at hand by first specifying a general domain from which these objects have to be selected (the family of all subsets of a set, the family of all $n$-tuples over a given set, etc.) and, then, by specifying additional conditions (constraints) these objects have to satisfy. In particular, constraint satisfaction problems are of this type.

Thus, to develop programs encoding solutions to such problems we might proceed in two steps. First, we develop an SLP program whose family of stable models encodes the general domain of candidate objects (all subsets, all sequences of length $n$, etc.). Next, we add to this program clauses representing constraints that must be enforced. 
In many applications involving subsets of some universe $U$ constraints are of the following form (here, $A$ and $B$ are two subsets of the same universe $U$ ):

$C(A, B)$ if a solution contains the set $A$, it must also contain at least one element from $B$ (or, in other words, a solution must not contain the set $A$ or must contain at least one element from the set $B$ )

Assume that $P$ is an SLP program whose stable models are subsets of $U$. Let $\mathcal{S}$ be a family of all stable models of $P$. To enforce the constraint $C(A, B)$ on sets in $\mathcal{S}$, that is, to construct a program whose stable models are exactly these stable models of $P$ that satisfy $C(A, B)$, it is enough to add to $P$ the clause (here $A=\left\{a_{1}, \ldots, a_{k}\right\}$ and $B=\left\{b_{1}, \ldots, b_{m}\right\}$ ):

$$
k_{C(A, B)}: \quad f \leftarrow a_{1}, \ldots, a_{k}, \operatorname{not}\left(b_{1}\right), \ldots, \operatorname{not}\left(b_{m}\right), \operatorname{not}(f)
$$

where $f$ is an atom not occurring in $P$. Formally, we have the following theorem.

Theorem 6.1 Let $P$ be a logic program and let $\mathcal{S}$ be the family of stable models of $P$. Then, for every constraint $C(A, B)$ where $A$ and $B$ are sets of atoms, $M$ is a stable model of $P \cup\left\{k_{C(A, B)}\right\}$ if and only if $M \in \mathcal{S}$ and $M$ satisfies $C(A, B)$.

As a corollary from Theorem 6.1, it follows that conjunctions of constraints of type $C(A, B)$ (that is, formulas in the conjunctive normal form) can be enforced by SLP programs consisting of clauses $k_{C(A, B)}$, for each conjunct $C(A, B)$. Moreover, the size of this SLP program is linear in the size of the CNF formula.

Theorem 6.1 is the first step towards a methodology of programming with SLP programs. A systematic study of this and other programming techniques appropriate in the case of SLP has not yet been conducted and has to be performed before SLP is used as a programming language. However, even this technique alone is quite powerful. We will now illustrate how it can be applied to encode several combinatorial problems.

First, we will revisit our "clique" problem. We assume that the graph is described by two lists of facts: vertex $(a)$ for all vertices $a$ of the graph (we will denote the set of vertices by $V$ ), and edge $(a, b)$, for all edges $\{a, b\}$ of the graph. We denote these lists of facts by $e d b_{c l q}(G)$. To specify cliques by an SLP program, one first needs to write a program specifying all subsets of the set of vertices of a graph. This can be accomplished, for instance, by the following two clauses:

(CLQ1) $\quad$ in $(X) \leftarrow \operatorname{vertex}(X), \operatorname{not}($ out $(X))$

(CLQ2) $\quad$ out $(X) \leftarrow \operatorname{vertex}(X), \operatorname{not}(\operatorname{in}(X))$

Stable models of the program consisting of clauses (CLQ1) and (CLQ2), and of facts $e d b_{c l q}(G)$ are of the form

$$
e d b_{c l q}(G) \cup\{i n(v): v \in K\} \cup\{\operatorname{out}(v): v \notin K\},
$$

where $K$ is a subset of the vertex set. Thus, the stable models of this program are in one-to-one correspondence to all subsets of the vertex set and can be regarded as their representations.

Next, we need to select those stable models that represent sets satisfying the clique condition: any two vertices in a clique are connected by an edge. This condition may be expressed as a constraint of the type $C(A, B)$ : if two vertices are in a clique then they are equal or they are connected with an edge. Thus, all these constraints (for all pairs of vertices) can be expressed by a single SLP clause: 


$$
\text { (CLQ3) } f \leftarrow \operatorname{vertex}(X), \operatorname{vertex}(Y), \text { in }(X), \text { in }(Y), \operatorname{not}(x=y), \operatorname{not}(\operatorname{edge}(x, y)), \operatorname{not}(f)
$$

By Theorem 6.1, the program consisting of facts $e d b_{c l q}(G)$, and clauses (CLQ1) - (CLQ3) has as its stable models precisely those sets of the form $e d b_{c l q}(G) \cup\{\operatorname{in}(v): v \in K\} \cup\{\operatorname{out}(v): v \notin K\}$, for which $K$ is a clique.

We will now further illustrate this approach by describing programs encoding the following problems:

1. Computing hamiltonian cycles in directed graphs,

2. Computing models of a propositional CNF formula,

For the first problem, we will need to represent directed graphs. Let $G$ be a directed graph with the set of vertices $V$ and with the set of directed edges $E$. We will represent $G$ by the following facts: vertex $(a)$, for all vertices $a \in V$, edge $(a, b)$, for all directed edges $(a, b) \in E$, and initialvtx $(a 0)$, for some vertex $a 0 \in V$. We will denote this representation of $G$ by $e d b_{h a m}(G)$.

Next consider the following clauses:

$$
\begin{aligned}
& \text { in }(V 1, V 2) \leftarrow \operatorname{edge}(V 1, V 2), \operatorname{not}(\operatorname{out}(V 1, V 2)) \\
& \operatorname{out}(V 1, V 2) \leftarrow \operatorname{edge}(V 1, V 2), \operatorname{not}(i n(V 1, V 2))
\end{aligned}
$$

These two clauses, together with the set of facts $e d b_{h a m}(G)$ define an SLP program whose stable models are of the form

$$
e d b_{\text {ham }}(G) \cup\{\operatorname{in}(a, b):(a, b) \in A\} \cup\{\text { out }(a, b):(a, b) \notin A\},
$$

for some set of edges $A$. Thus, these stable models represent all subsets of the set of edges of $G$. Those sets of edges that are hamiltonian cycles satisfy the following additional constraints: (1) if two edges of the cycle end in the same vertex, then these edges are equal, and (2) if two edges of the cycle start in the same vertex then these edges are equal. By Theorem 6.1, these constraints can be enforced by adding the following two clauses:

(HAM3) $\quad f \leftarrow i n(V 2, V 1), i n(V 3, V 1), \operatorname{not}(V 2=V 3), \operatorname{not}(f)$

(HAM4) $\quad f \leftarrow i n(V 1, V 2), i n(V 1, V 3), \operatorname{not}(V 2=V 3), \operatorname{not}(f)$

The stable models of the so expanded program are of the form $e d b_{h a m}(G) \cup\{\operatorname{in}(a, b):(a, b) \in$ $A\} \cup\{$ out $(a, b):(a, b) \notin A\}$, for some set of edges $A$ spanning in $G$ a set of vertex disjoint paths and cycles. Adding the following two clauses

(HAM5) $\quad$ reached $(V 2) \leftarrow i n(V 1, V 2), \operatorname{reached}(V 1)$

(HAM6) $\quad$ reached $(V 2) \leftarrow i n(V 1, V 2)$, initialvtx $(V 1)$

expands each of these stable models by the set of atoms reached $(a)$, for all these vertices $a$ that can be reached from the vertex $a 0$ by means of a nonempty sequence of edges that are "in" the model (note that we use this weaker notion of recursion here, that is still available in SLP). A stable model encodes a hamiltonian cycle if all vertices are reached. This constraint is enforced by the clause:

(HAM7) $\quad f \leftarrow \operatorname{not}(\operatorname{reached}(X)), \operatorname{not}(f)$ 
It follows that the program consisting of clauses (HAM1) - (HAM7) and of the facts in the set $e d b_{\text {ham }}(G)$ has as its stable models the sets of the form

$$
e d b_{\text {ham }}(G) \cup\{\operatorname{reached}(a): a \in V\} \cup\{\operatorname{in}(a, b):(a, b) \in A\} \cup\{\text { out }(a, b):(a, b) \notin A\},
$$

for which $A$ is the set of the edges of a hamiltonian cycle in $G$.

For the satisfiability problem, we need to represent CNF formulas. Consider a CNF formula $\varphi$ with the set of clauses $\mathcal{C}$ and the set of variables $V$. The formula $\varphi$ will be represented by several lists of facts: $\operatorname{var}(a)$, for each $a \in V$, clause $(c)$, for each clause $c \in \mathcal{C}, \operatorname{pos}(c, v)$, for each variable $v$ and clause $c$ such that $v$ appears positively in $c$, and $n e g(c, v)$, for each variable $v$ and clause $c$ such that $v$ appears negatively in $c$. This specification of a CNF formula $\varphi$ will be denoted by $e d b_{\text {sat }}(\varphi)$.

Consider now the following clauses:

$$
\begin{aligned}
& \operatorname{true}(X) \leftarrow \operatorname{var}(X), \operatorname{not}(\text { false }(X)) \\
& \text { false }(X) \leftarrow \operatorname{var}(X), \operatorname{not}(\operatorname{true}(X))
\end{aligned}
$$

These two clauses generate all possible truth assignments to variables in $V$. More formally, the program consisting of $e d b_{s a t}(\varphi)$ and clauses (SAT1) and (SAT2) has as its stable models the sets of the form $e d b_{\text {sat }}(\varphi) \cup\{\operatorname{true}(v): v \in U\} \cup\{$ false $(v): v \in V \backslash U\}$, where $U$ is a subset of $V$. Thus, they represent the set of all valuations. The next two clauses

$$
\begin{aligned}
& \operatorname{sat}(C) \leftarrow \operatorname{var}(X), \text { clause }(C), \operatorname{true}(X), \operatorname{pos}(C, X) \\
& \operatorname{sat}(C) \leftarrow \operatorname{var}(X), \operatorname{clause}(C), \text { false }(X), \operatorname{neg}(C, X)
\end{aligned}
$$

simply define when a clause is satisfied and add to each stable model the set of clauses that are true in the valuation represented by this model. Formula $\varphi$ is satisfiable if there is a valuation which makes all clauses true. This requirement can be enforced by adding the clause

$$
f \leftarrow \operatorname{clause}(C), \operatorname{not}(\operatorname{sat}(C)), \operatorname{not}(f)
$$

It follows that $M$ is a stable model of the program consisting of clauses (SAT1) - (SAT5) and of the facts in the set $e d b_{s a t}(\varphi)$ if and only if

$$
M=e d b_{\text {sat }}(\varphi) \cup\{\operatorname{sat}(c): c \in \mathcal{C}\} \cup\{\operatorname{true}(v): v \in U\} \cup\{\operatorname{false}(v): v \in V \backslash U\},
$$

where $U \subseteq V$ is a (propositional) model of $\varphi$.

The encodings presented so far are uniform. That is, input data to a problem is encoded as a collection of facts and the constraints defining the problem as clauses (usually with variables) with the latter part not depending on a particular input. These two parts correspond well to the extensional and intensional components of a DATALOG $\urcorner$ program. However, other encodings are also possible (and often easier to come up with).

To make the point, let us again consider the satisfiability problem. As before, consider a CNF formula $\varphi$ with a set of variables $V$ and a set of clauses $\mathcal{C}$. This time we will represent valuations as subsets of $V$. It turns out that there is a very simple propositional encoding of the satisfiability problem. First, note that the clauses

$\left(\mathrm{SAT}^{\prime} 1\right) \quad$ in $(v) \leftarrow \operatorname{not}($ out $(v))$

$\left(\mathrm{SAT}^{\prime} 2\right) \quad \operatorname{out}(v) \leftarrow \operatorname{not}(i n(v))$ 
(where in both $\left(\mathrm{SAT}^{\prime} 1\right)$ and $\left(\mathrm{SAT}^{\prime} 2\right) v$ ranges over $V$ ), specify all subsets of $V$. That is, the stable models of the program consisting of all clauses $\left(\mathrm{SAT}^{\prime} 1\right)$ and $\left(\mathrm{SAT}^{\prime} 2\right)$ are precisely the sets of the form $\{i n(a): a \in M\} \cup\{\operatorname{out}(a): a \in V \backslash M\}$, where $M$ is a subset of $V$. Now, for each clause

$$
c: \quad \neg a_{1} \vee \ldots \vee \neg a_{k} \vee b_{1} \vee \ldots \vee b_{m}
$$

of $\varphi$, add to the program the SLP clause

$$
f \leftarrow a_{1}, \ldots, a_{k}, \operatorname{not}\left(b_{1}\right), \ldots, \operatorname{not}\left(b_{m}\right), \operatorname{not}(f)
$$

Since a set of atoms satisfies clause $c$ if and only if it satisfies the constraint $C\left(\left\{a_{1}, \ldots, a_{k}\right\}\right.$, $\left.\left\{b_{1}, \ldots, b_{m}\right\}\right)$, it follows that the program consisting of all clauses $\left(\mathrm{SAT}^{\prime} 1\right)-\left(\mathrm{SAT}^{\prime} 3\right)$ has as its stable models sets of the form $\{i n(a): a \in M\} \cup\{\operatorname{out}(a): a \in V \backslash M\}$, where $M$ is a model of $\varphi$.

Let us emphasize that the encodings discussed in this section are not unique. Two satisfiability encodings given here constitute but one example. Further, a different encoding of the hamiltonian cycle problem can be found in Nie98 and more encodings (as propositional default theories and logic programs) for several combinatorial problems were given in [CMMT98]. These encodings may have different computational properties. In particular, the second encoding of satisfiability, even though it is not uniform and requires that a separate program be created for each satisfiability instance, may actually be better suited for processing. Thus, the issue of the programming methodology likely to result in programs whose stable models can be quickly computed is very important. It has not been studied yet but must receive significant attention if stable logic programming is to become a practical problem solving tool.

\section{Uniform control in SLP}

As we have seen in the earlier sections, SLP programs can specify a wide class of search and decision problems. In addition, they do so in a declarative fashion by modeling, in a direct way, constraints defining a problem at hand. Thus, SLP programs are well suited to represent the "logic" part in Kowalski's "algorithm = logic + control" phrase.

In order for the stable logic programming to serve as an effective computational problem solving tool (and not only as a knowledge representation formalism), we need to develop the other component of the Kowalski's equation - a uniform control. Since, in stable logic programming, problems are encoded by SLP programs and solutions correspond to stable models, this uniform control must consist of algorithms to process SLP programs and compute (or decide the existence of) their stable models.

Several such algorithms were proposed in the recent years. In particular, algorithms were proposed to decide the existence of stable models of a propositional logic program $P$, and to compute one (or all) stable models of $P$, if they exist. Algorithms that decide the membership of an atom in some or all stable models were also developed [NS96, CMT96, ELM ${ }^{+} 97$, ADN97].

These algorithms employ a backtracking search through the space of all subsets of the Herbrand base of the program (the collection of all propositional letters that occur in the program). They also use a variety of search space pruning techniques. Some of these techniques 
rely on a generalization of the concept of stratification CMT96. Other methods use wellfounded semantics in a way unit propagation is used in Davis-Putnam procedure for computing models of CNF formulas [NS96.

Notice that since there are no function symbols in the language of SLP, the Herbrand universe, the Herbrand base and the grounding of an arbitrary (that is, not necessarily propositional) finite SLP program are finite. There are straightforward algorithms to produce the (finite) ground version of a finite SLP program. More sophisticated algorithms, minimizing the size of the ground program while preserving the stable models, were proposed recently in NS96, Cho96. Thus, algorithms to compute stable models of finite propositional programs can be used with arbitrary finite SLP programs, too (and termination is guaranteed).

These algorithms led to implementations of several systems for processing SLP programs and for computing their stable models. Among them are smodels NS95, NS96, DeReS CMT96 and the system described in [ELM ${ }^{+}$97]. Any of these systems can be used as a control mechanism for the stable logic programming environment and transforms SLP from a knowledge representation formalism into a computational programming tool.

Thus, in the transition from Horn logic programming to SLP not only the semantics and the methodology of programming changes. The control has to change, too. Instead of SLD resolution, the basis for the control mechanism of SLP is provided by backtracking search algorithms for search spaces of subsets of a given finite set (Herbrand base of a finite SLP program).

The stable logic programming became a viable proposal for a new logic programming system with recent advances in algorithms for computing stable models and subsequent implementations of these algorithms [NS96, CMT96, ELM ${ }^{+}$97]. Even though comprehensive studies of these implementations have yet to be performed, available results provide reasons for optimism. For some classes of programs, systems such as smodels [NS96] and DeReS [CMT96 can successfully process programs with tens of thousands of clauses. In addition, as reported in Nie98, smodels can successfully compete on a class of planning problems with special purpose planners (for more comprehensive discussion of applications of logic programming to planning see [Lif98] and a collection of papers referred to there). It is clear that with the performance of the systems computing stable models improving, the attractiveness of SLP as a computational tool will grow.

Despite a significant progress and the existence of systems such as smodels and DeReS, much more work on algorithms for computing stable models is needed in order to obtain acceptable performance. There are several open problems such as development of new and more powerful pruning techniques and study of probabilistic algorithms for stable model computation.

\section{Conclusions and future directions}

In the paper we discussed the stable model semantics as the foundation of a computational logic programming system different from Horn logic programming. This system, the stable logic programming or SLP, shares with other logic programming systems their key feature: the separation of logic from control. However, despite the fact that the stable model semantics has its roots in the efforts to extend the principles of Horn logic programming to the case of programs with negation, the stable logic programming in several aspects differs significantly from standard logic programming systems. 
1. In the SLP programs are assigned a collection of intended models rather than a single intended model as in Horn logic programming, stratified logic programming or logic programming with well-founded semantics.

2. Since there are no function symbols in the language, higher-order objects are represented in the SLP as stable models of programs rather than as ground terms of the Herbrand universe, as it is the case in Horn logic programming and other similar systems.

3. SLP programs are interpreted as sets of constraints on objects to be computed unlike in Horn logic programming where clauses model recursive definitions rather than constraints.

4. The control mechanism of SLP is no longer resolution-based. Instead, the uniform control of SLP consists of backtracking search algorithms for computing stable models of programs.

5. The SLP has lower expressive power than standard logic programming systems. While it may seem to be a limitation, the class of problems that can be solved in the SLP is still quite wide and includes all decision problems from NP and many search and constraint satisfaction problems of importance in artificial intelligence and operations research.

We believe that the perspective of stable logic programming presented in the paper certainly warrants further investigations. We will now outline several interesting research directions.

Let us start with the following fundamental question: why to use the stable logic programming and not simply propositional logic. Indeed, a finite collection of clauses (possibly with variables but not with function symbols) together with a finite collection of facts can be viewed as a representation of a finite propositional formula in the conjunctive normal form. Consequently, it can be viewed as an encoding of the family of models of this formula (in the same way in which an SLP program represents the family of its stable models - a key observation underlying stable model programming). Moreover, since propositional satisfiability problem is NP-complete, all decision problems in NP can be reduced in polyniomial time to satisfiability testing (and solved by means of programs such as Davis-Putnam procedure for satisfiability testing). Finally, constraints of the type $C(A, B)$ can easily be encoded as clauses.

Is there then a real need to resort to logic programming? This is a challenging open problem. We believe the answer is positive but cannot offer any rigorous argument in support of the claim. In our opinion, the advantage of stable logic programming stems from the following two properties of stable models. First, they are minimal. Thus, when dealing with optimization problems minimality comes for free with the stable model semantics. Second, they are grounded - facts are included in stable models only if they can be justified. Furthermore, a comparison between existing encodings of combinatorial problem shows that in many cases encodings in terms of logic programs are more concise than those in terms of satisfiability (possibly due to the groundedness property of stable models). In particular, we believe that the most concise encodings of the existence of a hamiltonian cycle problem as the problem of the existence of a stable model of a logic program are asymptotically more more concise than similar encodings as the problem of the existence of a satisfying valuation of a CNF formula.

Developing a formal setting to compare stable logic programming with propositional logic and providing a rigorous account of advantages and disadvantages of both approaches are important general theoretical challenges. 
The second important research direction is the development of a systematic study of the methodology for developing SLP programs. Some initial steps in this direction were presented in Section 6. There is a potential trade-off here. On one hand, one of the most important objectives is ease of program development. On the other hand, we want our programs to run fast.

Third, despite the recent successes with implementing systems based on the stable model semantics, there is still much to be done. So far, performance studies for the existing systems have been rather ad hoc. More comprehensive experimental studies are needed that will give insights into the computational nature of stable models and will lead to faster algorithms. To support such studies one needs benchmarking systems. One step in this direction is TheoryBase, a system described in [CMMT95, CMMT98].

Next, the SLP seems to be especially well suited for dealing with constraint satisfaction problems. Thus, it is important to extend the language of the SLP so that important classes of constraints involving arithmetic operations and relations became easier to model. Possibility of incorporating the SLP into existing constraint solving systems is another important problem.

Finally, there is already evidence that the SLP can be a useful tool in solving planning problems Nie98]. Studying applicability of the SLP paradigm to other classical problems of artificial intelligence and operations research may provide additional motivation to focusing on this approach and may gain badly needed recognition to logic programming.

\section{References}

[AB90] K. Apt and H.A. Blair. Arithmetical classification of perfect models of stratified programs. Fundamenta Informaticae, 12:1-17, 1990.

[ABW88] K. Apt, H.A. Blair, and A. Walker. Towards a theory of declarative knowledge. In J. Minker, editor, Foundations of deductive databases and logic programming, pages 89-142, Los Altos, CA, 1988. Morgan Kaufmann.

[ACY91] F. Afrati, S. Cosmodakis, and M. Yannakakis. On DATALOG vs. polynomial time. In Proceedings of PODS'91, pages 13-25, 1991.

[ADN97] C. Aravindan, J. Dix, and I. Niemelä. Dislop: Toward a disjunctive logic programming system. In Proceedings of the 4th International Conference on Logic Programming and Nonmonotonic Reasoning, pages 341-352, 1997. Springer LN in Computer Science 1265.

[AHV95] S. Abiteboul, R. Hull, and V. Vianu. Foundations of Databases. Addison-Wesley Publishing Company, 1995.

[AN78] H. Andreka and I. Nemeti. The generalized completeness of Horn predicate logic as a programming language. Acta Cybernetica, 4:3-10, 1978.

[AvE82] K.R. Apt and M.H. van Emden. Contributions to the theory of logic programming. Journal of the ACM, 29:841-862, 1982.

[BF91] N. Bidoit and C. Froidevaux. Negation by default and unstratifiable logic programs. Theoretical Computer Science, 78:85-112, 1991. 
[BMS95] H.A. Blair, W. Marek, and J. Schlipf. The expressiveness of locally stratified programs. Annals of Mathematics and Artificial Intelligence, 15:209-229, 1995.

[Cho96] P. Cholewinski. Automated reasoning with Default Logic. PhD thesis, University of Kentucky, 1996. Ph.D. Thesis.

[CKPR73] A. Colmerauer, H. Kanoui, R. Pasero, and P. Roussel. Un systeme de communication homme-machine en francais. Technical report, University of Marseille, 1973.

[CL73] C.-L. Chang and C.-T. Lee. Symbolic Logic and Mechanical Theorem Proving. Academic Press, 1973.

[Cla78] K.L. Clark. Negation as failure. In H. Gallaire and J. Minker, editors, Logic and data bases, pages 293-322. Plenum Press, 1978.

[CMMT95] P. Cholewiński, W. Marek, A. Mikitiuk, and M. Truszczyński. Experimenting with nonmonotonic reasoning. In Proceedings of the 12th International Conference on Logic Programming, pages 267-281. MIT Press, 1995.

[CMMT98] P. Cholewiński, W. Marek, A. Mikitiuk, and M. Truszczyński. Programming with default logic. Submitted for publication, 1998.

[CMT96] P. Cholewiński, W. Marek, and M. Truszczyński. Default reasoning system deres. In Proceedings of KR-96, pages 518-528. Morgan Kaufmann, 1996.

[CP98] M. Cadoli and L. Palipoli. Circumscribing datalog: expressive power and complexity. Theoretical Computer Science, 193:215-244, 1998.

[CPSV98] M. Cadoli, L. Palipoli, A. Schaerf, and D. Vasile. Np-spec: An executable specification language for solving all problems in np. Unpublished manuscript, 1998.

$\left[\mathrm{ELM}^{+}\right.$97] T. Eiter, N. Leone, C. Mateis, G. Pfeifer, and F. Scarcello. A deductive system for non-monotonic reasoning. In Proceedings of the 4 th International Conference on Logic Programming and Nonmonotonic Reasoning, pages 363-374, 1997. Springer LN in Computer Science 1265.

[GJ79] M.R. Garey and D.S. Johnson. Computers and intractability; a guide to the theory of NP-completeness. W.H. Freeman, 1979.

[GL88] M. Gelfond and V. Lifschitz. The stable semantics for logic programs. In R. Kowalski and K. Bowen, editors, Proceedings of the 5th International Symposium on Logic Programming, pages 1070-1080, Cambridge, MA, 1988. MIT Press.

[Her30] J. Herbrand. Recherches sur la théorie de la démonstrations. PhD thesis, Paris, 1930 .

[Kam97] M. Kaminski. A note on stable semantics for logic programs. Technion, Israel Institute of Technology, 11pp., 1997. 
[Kow74] R. Kowalski. Predicate logic as a programming language. In Proceedings of IFIP 74, pages 569-574, Amsterdam, 1974. North Holland.

[Kow79] R. Kowalski. Logic for Problem Solving. North Holland, Amsterdam, 1979.

[Lif98] V. Lifschitz. Action languages, answer sets and planning. unpublished manuscript, 1998.

[Llo84] J. Lloyd. Foundations of logic programming. Berlin: Springer-Verlag, 1984.

[Lov78] D. Loveland. Automated Theorem Proving: A Logical Basis. North Holland, 1978.

[MNR92] W. Marek, A. Nerode, and J. B. Remmel. The stable models of predicate logic programs. In Proceedings of international joint conference and symposium on logic programming, Boston, MA, 1992. MIT Press.

[MT89] W. Marek and M. Truszczyński. Stable semantics for logic programs and default theories. In E.Lusk and R. Overbeek, editors, Proceedings of the North American Conference on Logic Programming, pages 243-256. MIT Press, 1989.

[MT91] W. Marek and M. Truszczyński. Autoepistemic logic. Journal of the ACM, 38:588619, 1991.

[MT93] W. Marek and M. Truszczyński. Nonmonotonic logics; context-dependent reasoning. Springer-Verlag, 1993.

[MW88] D. Maier and D. S. Warren. Computing with logic. Logic programming with Prolog. The Benjamin/Cummings Publishing Company, Inc., 1988.

[Nie98] I. Niemelä. Logic programs with stable model semantics as a constraint programming paradigm. In I. Niemelä and T. Schaub, editor, Proceedings of the Workshop on Computational Aspects of Nonmonotonic Reasoning, pages 72-79, 1998.

[NS95] I. Niemelä and P. Simons. Evaluating an algorithm for default reasoning. In Proceedings of the IJCAI-95 Workshop on Applications and Implementations of Nonmonotomic Reasoning Systems, 1995.

[NS96] I. Niemelä and P. Simons. Efficient implementation of the well-founded and stable model semantics. In Proceedings of JICSLP-96. MIT Press, 1996.

[Prz88] T. Przymusiński. On the declarative semantics of deductive databases and logic programs. In Foundations of deductive databases and logic programming, pages 193-216, Los Altos, CA, 1988. Morgan Kaufmann.

[Prz90] T. Przymusinski. Stationary semantics for disjunctive logic programs. Technical report, University of Texas at El Paso, 1990.

[Rei80] R. Reiter. A logic for default reasoning. Artificial Intelligence, 13:81-132, 1980.

[Rob65] J.A. Robinson. Machine-oriented logic based on resolution principle. Journal of the ACM, 12:23 - 41, 1965. 
[RRS $\left.{ }^{+} 95\right]$ P. Rao, I.V. Ramskrishnan, K. Sagonas, T. Swift, and D. S. Warren. Efficient tabling mechanisms for logic programs. In Proceedings of the 12th International Conference on Logic Programming, Cambridge, MA, 1995. MIT Press.

[RRS $\left.{ }^{+} 97\right]$ P. Rao, I.V. Ramskrishnan, K. Sagonas, T. Swift, D. S. Warren, and J. Freire. XSB: A system for efficiently computing well-founded semantics. In Proceedings of LPNMR'97, pages 430-440. Berlin: Springer-Verlag, 1997. Lecture Notes in Computer Science, 1265.

[Sch95] J. Schlipf. The expressive powers of the logic programming semantics. Journal of the Computer Systems and Science, 51:64 - 86, 1995.

[Smu68] R.M. Smullyan. First-order logic. Berlin: Springer-Verlag, 1968.

[Ull88] J.D. Ullman. Principles of Database and Knowledge-Base Systems. Computer Science Press, Rockville, MD, 1988.

[VRS91] A. Van Gelder, K.A. Ross, and J.S. Schlipf. Unfounded sets and well-founded semantics for general logic programs. Journal of the ACM, 38:620 - 650, 1991. 